\title{
Risk factors associated with diabetic foot disease in diabetes patients: First national study in Oman
}

\author{
Abdullah Rashid Al Jabri', Adhra Al Mawali2,3,*
}

\author{
College of Medicine, Sultan Qaboos University, Muscat, Oman. \\ ${ }^{2}$ Center of Studies and Research, Ministry of Health, Muscat, Oman. \\ ${ }^{3}$ Strategic Research Program for Non-Communicable Diseases, The Research Council, Muscat, Oman. \\ *Correspondence to: Adhra Al Mawali (E-mail: adhra.almawali@gmail.com) \\ (Submitted: 16 January 2021 - Revised version received: 14 February 2021 - Accepted: 25 February 2021 - Published online: 26 April 2021)
}

\begin{abstract}
Objective The aim of this study is to identify the correlation between multiple risk factors in the development of diabetic foot disease (DFD) in Oman. It also aims to identify the effect of having multiple significant risk factors on the disease progression, and to explore which risk factor shows the highest correlation with disease development.

Methods A retrospective case-control study was conducted with 100 patients and 200 controls. Data of the participants were extracted from hospital's Electronic Patient Record System (Alshifa) from 2000 to 2018. Chi-square, Fisher exact test, Odds Ratio and Multiple regression analysis were used to determine the significance of various risk factors.

Results Having a $\mathrm{HbA} 1 \mathrm{c}>7$, body mass index $>30 \mathrm{~kg} / \mathrm{m}^{2}$, and blood pressure over 140/90 mmHg showed a strong correlation with the development of DFD. Other risk factors such as age of diabetes, gender, total blood cholesterol, triglyceride levels, LDL, and HDL did not show any significant correlation with DFD.

Conclusions Risk factors for DFD are highly prevalent in our society, controlling these risk factors could minimize the morbidity and the mortality related to this disease as well as reducing the economic impact related to it. Proper education for those at a higher risk could play an important role in the control of this disease.

Keywords Diabetic foot, Risk factors, Body mass index, Oman
\end{abstract}

\section{Advances in Knowledge}

- This is the first study to assess the risk factors of DFD in Oman.

- The study revealed high incidents of uncontrolled risk factors in Oman.

- It opens the door for future articles to study this disease and explore more about it.

\section{Application to Patient Care}

- This study gives the community chance to identify DFD risk factors and by that it is possible to prevent getting high numbers of new DFD cases by controlling these modifiable risk factors.

- Creating a new management technique to control these risk factors.

- Increased public awareness of DFD risk factors and its substantial impact on the incidence and outcomes of the disease.

\section{Introduction}

One of the most difficult challenges that faces modern medicine is chronic diseases, ${ }^{1}$ and diabetes mellitus (DM) is one of the devastating conditions with many complications, the World Health Organization (WHO) estimated that the global prevalence of diabetes among adults (above 18 years) was $8.5 \%$ in $2014 .^{2}$ Based on the International Diabetes Federation (IDF), the prevalence of diabetes in the Middle East and North Africa (MENA) was $9.6 \%$ in the population with age from 20 to 79 in $2017 .^{3}$ The IDF predict that the prevalence will increase to $12.1 \%$ in $2045 .{ }^{3}$ Oman is of no exception in this regard. 71,625 patients of the Omani population are suffering from diabetes based on a Ministry of Health $(\mathrm{MoH})$ records in 2011. ${ }^{4}$ The annual health report of Ministry of Health $(\mathrm{MoH})$ in 2016 showed that the number of diabetic patients in Oman was 89,246 for all those who are registered in $\mathrm{MoH}$ Al Shifa system. ${ }^{5}$ The recent STEPs survey for non-communicable diseases (NCD) 2107 revealed that approximately $14.5 \%$ of the Omani population are diabetic. ${ }^{6} 86.6 \%$ were identified by the health system and $13.4 \%$ were newly diagnosed during the survey. ${ }^{6}$

Diabetes is a very serious condition if not controlled. ${ }^{2}$ This disease can lead to various consequences. There are many manifestations of diabetic complications, but the most important ones are cardiovascular diseases, neuropathy, nephropathy, retinopathy, skin conditions, hearing impairment, Alzheimer's disease and diabetic food disease (DFD).

DFD is a disastrous complication of diabetes, and it is defined as foot ulcers associated with peripheral arterial disease and neuropathy which can lead to development of gangrene that may eventually require lower limb amputation. ${ }^{7}$ The prevalence of DFD has a large variation depending on the region and the income of the country. ${ }^{3}$ The average prevalence of DFD worldwide is $6.4 \%$ among diabetic patients. ${ }^{3}$ In the MENA region, the prevalence of DFD in Saudi Arabia was $4.3 \%$, while it was a bit higher in Jordan and Algeria with prevalence of $5 \%$ and $11.9 \%$ respectively.

DFD is a serious complication and it can impact the patient's quality of life. ${ }^{7}$ Based on IDF, those who have diabetes are at risk of requiring surgical amputation 10-20 times more than those who do not have it. ${ }^{3}$ Almost half of the lower limbs amputation in Oman from 2003 to 2013 are diabetes-related and it is anticipated to rise in the upcoming years. ${ }^{911}$ In 2007, one-third of diabetes treatment cost was spent on foot ulcers, 
and this shows that DFD is a true burden from the economic aspect worldwide. ${ }^{3}$ There are many risk factors that have been shown to have a direct relation in developing DFD. These risk factors are age, gender, smoking, diabetes duration, hypertension, neuropathy, peripheral vascular disease and poor glycemic control. ${ }^{12}$ Al-Rubeaan et al in Saudi Arabia showed that DFD is associated with certain risk factors such as duration of diabetes, hypertension, and poor glucose control. ${ }^{12}$

There is a paucity in studies related to DFD as there are only two studies conducted in Oman since 1991 about DFD; and they did not investigate the different risk factors associated with DFD. ${ }^{13}$ There was a call from Oman Medical Journal for more research in DFD in April 2017 in this area. ${ }^{13}$ Thus, to the best of our knowledge, this is the first study in Oman that focuses mainly on the risk factors associated with development of DFD. This study aims to identify the correlation between multiple risk factors in the development and progression of DFD in Oman.

\section{Methods}

A retrospective case-control study was conducted from December 2018 to March 2019. This study covered all MoH health centers from all Governorates of Oman. Data were collected from the case records of those who were clinically diagnosed to have DFD due to Type 2 DM from 2000 to 2018, almost two decades regardless of their onset of diabetes. The population surveyed adults aged 20 years or older, men and women, as well as nationals and non-national residents. Those younger than 20 years old, tourists, and persons from labor camps were excluded. Patient's anonymity was maintained to ensure patient confidentiality. True crypt (program used to keep data protected) was used as a safety measure to ensure confidentiality. All retrieved case files were studied thoroughly to find out all the relevant data related to DFD patients including their presence of foot ulcers, date of diagnosis, onset of disease, age, gender, HbAc1 (levels of glycosylated hemoglobin), body mass index (BMI), LDL, HDL, triglyceride, and total blood cholesterol before getting DFD. Average of three consecutive results was taken for the highest representation of the patients controlling status. Other risk factors that were planned to be included are smoking status, social status, and educational status but unfortunately these risk factors were not available for most of the patients.

\section{Sample size calculation}

Sample size calculation for this study was done using Epi info software. A 95\% confidence level and a power of $90 \%$ was used with a ratio of controls to cases of two. The sample size for the study group (cases) was calculated to be 100 patients. A total of 100 DM case files with DFD and 200 DM controls without DFD were studied from December 2018 to March 2019, using case files extracted from 79 medical centers, representing all Governorates of Oman.

\section{Case selection strategy}

To minimize bias and to ensure generalizability, the patients included in the study were chosen through random sampling by choosing random case number from the records (regardless of date of diagnosis, age, and gender). Microsoft excel was used to get random sequence of patients according to their number from each health center.

\section{Ethical approval}

Prior to its initiation, the ethical approval was obtained from Research and Ethical Review \& Approve Committee (RERAC) of $\mathrm{MoH}$.

\section{Inclusion criteria}

All samples (cases and controls) were type $2 \mathrm{DM}$, they should be above 20-year-old and they must have a patient record in $\mathrm{MOH}$ system (Alshifa). The selection criteria for cases is that they must have DFD while controls do not have DFD.

\section{Exclusion criteria}

Patients with type $1 \mathrm{DM}$ and those who are younger than 20 years were excluded. Diabetes unrelated ulcers and amputations were excluded. Pregnant women who have gestational diabetes were excluded as well. In addition, those who have genetic diseases such as sickle cell disease and rheumatoid arthritis were eliminated because of the impact of these diseases on ulcers formation. These exclusion criteria were applied for cases and controls. The inclusion and exclusion criteria were strictly followed to avoid any confusion that could affect the quality of the results.

\section{Statistical analysis}

Statistical analysis was calculated using the SPSS 23.0 software. Further analysis using Chi-square test was done to assess the correlation of multiple risk factors and DFD. Multiple risk factors such as age of diabetes, gender, hypertension, BMI, total blood cholesterol, triglyceride levels, LDL, HDL, and poor glycemic control were studied. Odds ratio were also calculated. Reference ranges of the international standard of WHO was used for the risk factors. Data were safely stored and encrypted using TrueCrypt software to ensure confidentiality and integrity of patient records.

\section{Results}

Three-hundred patients (100 Cases and 200 Controls) were included in this study from all governorates of Oman.

Mean age of the population was calculated and the gender distribution was demonstrated in Fig. 1 and Table 1.

Diabetic males mean age was slightly less (56) and it was very near to diabetic females mean age which was 58 years (Table 3). Females who are suffering from DFD had mean age of 64 and the number was higher in DFD male patients with a mean age of 66 (Table 2).

\section{Poorly controlled blood glucose (HbA1C)}

Among patients of DFD 91\% ( $n=91)$ had poorly controlled blood sugar while only $9 \%(n=9)$ had controlled blood glucose (Table 3). Table 3 shows that $52 \%(n=104)$ of diabetic patients had poorly controlled blood sugar and $48 \%(n=96)$ were having a good control. Poorly controlled diabetic patients showed a significant association in developing DFD. DM patients with DFD (cases) are 9.3 times more likely to have elevated HbA1C than the controls without DFD (univariate analysis $P$-value $<0.001$ ) (multivariate analysis $P$-value $<0.001$ ) (Table 4 ).

\section{Body mass index}

The second risk factor studied is BMI, which also showed a big variation between cases and controls. Obesity appeared in $40 \%$ 


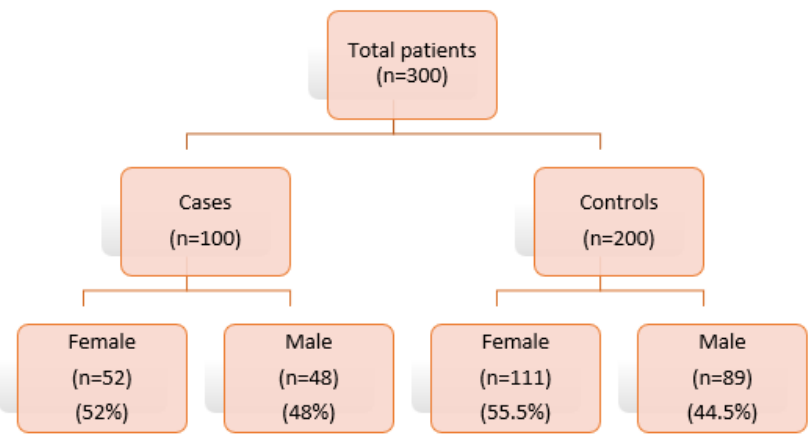

Fig. 1 Patients demographic characteristics.

Table 1. Age at onset of diabetes among DM patients and DFD patients.

\begin{tabular}{lcc} 
DFD patients (Cases) age at & $20-44$ & $26 \%(n=26)$ \\
onset diabetes & $45-65$ & $64 \%(n=64)$ \\
& $>65$ & $10 \%(n=10)$ \\
& $20-44$ & $37.5 \%(n=75)$ \\
Diabetic patients (Controls) age & $45-65$ & $54.5 \%(n=109)$ \\
at onset of diabetes & $>65$ & $8 \%(n=16)$ \\
& & \\
\hline
\end{tabular}

Table 2. Mean age of each sex among DM patients and DFD patients.

\begin{tabular}{llc}
\hline Diabetic Patients & Female & Male \\
(Controls) Mean age & 58 years & 56 years \\
DFD Patients & Female & Male \\
(Cases) Mean age & 64 years & 66 years \\
\hline
\end{tabular}

$(\mathrm{n}=80)$ of the controls and $60 \%(\mathrm{n}=120)$ were non-obese. On the other hand, $62 \%(n=62)$ of DFD patients were obese and $38 \%(n=38)$ were non-obese. Our study demonstrated that being an obese is a significant risk factor that is associated with DFD. DM patients with DFD are 3.2 times more likely to be obese than controls without DFD (univariate analysis $P$-value $<0.001)$ (multivariate analysis $P$-value $=0.034)($ Table 4$)$.

\begin{tabular}{|c|c|c|c|c|}
\hline & \multirow{2}{*}{$P$ value } & \multirow{2}{*}{$\begin{array}{l}\text { Odds } \\
\text { ratio }\end{array}$} & \multicolumn{2}{|c|}{$95 \%$ C.I. for Odds ratio } \\
\hline & & & Lower & Upper \\
\hline $\mathrm{Hba1c}$ & .000 & 8.6 & 18.9 & 4.0 \\
\hline Blood pressure & .000 & 4.5 & 8.1 & 2.5 \\
\hline BMI & .034 & 1.9 & 3.3 & 1.1 \\
\hline Total cholesterol & .510 & 1.3 & 3.0 & 0.6 \\
\hline Triglyceride & .800 & 1.1 & 2.0 & 0.6 \\
\hline $\mathrm{HDL}$ & .553 & 1.4 & 4.1 & 0.5 \\
\hline LDL & .871 & 0.9 & 2.0 & 0.4 \\
\hline Constant & .028 & 4.004 & & \\
\hline
\end{tabular}

\section{Blood pressure}

Table 3 shows that blood pressure (BP) was high in $78 \%(n=78)$ of DFD patients while only $28 \%(n=28)$ had controlled BP. Percentages were significantly different in diabetic patients, $66.5 \%(n=135)$ had controlled BP and 33.5\% $(n=70)$ were not. $\mathrm{BP}$ was a significant risk factor DM patients with DFD are 5.3 times more likely to have high blood pressure than controls without DFD (univariate analysis $P$-value $<0.001$ ) (multivariate analysis $P$-value $<0.001$ ) (Table 4 ).

\section{Gender}

Females were slightly more in both cases $52 \%(\mathrm{n}=52)$ and control $55.5 \%(\mathrm{n}=111)$ when compared to males in both cases $48 \%(n=48)$ and controls $44.5 \%(n=89)$. But the difference was not statistically significant (Fig. 1) ( $p$-value $=0.56)$.

\section{Age at onset of diabetes}

Age at onset of diabetes was divided into three groups (20-44, $45-65,>65)$. Among DFD patients, age at onset of diabetic patients showed the following: $26 \%(n=26)$ had diabetes in the age between 20 and 44 years and $64 \%(n=64)$ got the disease in the age between 45 and 65 years, while only $10 \%(n=10)$ acquired the disease above 65 years old. On the other side, diabetic patient's age showed the following: $37.5 \%(n=75)$ of them had diabetes in the age between 20 and 44 years and

\begin{tabular}{|c|c|c|c|c|c|c|c|c|}
\hline & \multicolumn{3}{|c|}{ T2DM patients } & \multicolumn{3}{|c|}{ DFD patients } & \multirow{2}{*}{$P$ valuea } & \multirow{2}{*}{ Odds ratio } \\
\hline & Total & Normal & Abnormal & Total & Normal & Abnormal & & \\
\hline Total number & 200 & & & 100 & & & & \\
\hline $\mathrm{HbA1c}$ & 200 & $96(48 \%)$ & $104(52 \%)$ & 100 & $9(9 \%)$ & $91(91 \%)$ & $<0.001$ & 9.3 \\
\hline BMI & 200 & $120(60 \%)$ & $80(40 \%)$ & 100 & $32(32 \%)$ & $68(68 \%)$ & $<0.001$ & 3.2 \\
\hline Blood pressure & 200 & 133(66.5\%) & 67(33.5\%) & 100 & $28(28 \%)$ & $78(78 \%)$ & $<0.001$ & 5.5 \\
\hline Total cholesterol & 200 & 158(79\%) & $42(21 \%)$ & 100 & 70(70\%) & $30(30 \%)$ & 0.09 & 1.6 \\
\hline Triglyceride & 200 & $136(68.5 \%)$ & $64(31.5 \%)$ & 100 & $57(57 \%)$ & $43(43 \%)$ & 0.06 & 1.6 \\
\hline HDL & 200 & 196(98\%) & $4(2 \%)$ & 100 & 98(98\%) & $2(2 \%)$ & 1 & 1 \\
\hline LDL & 200 & $126(63 \%)$ & $74(37 \%)$ & 100 & $58(58 \%)$ & $42(42 \%)$ & 0.40 & 1.2 \\
\hline
\end{tabular}


$54.5 \%(n=109)$ got the disease in the age between 45 and 65 while only $8 \%(n=16)$ acquired the disease in the age above 65 $(p$-value $=0.14)($ Table 1$)$.

\section{Total cholesterol}

Total blood cholesterol was also studied as a risk factor for DFD and the percentages was as the following: 70\% $(n=70)$ of DFD patients had a normal total cholesterol and $30 \%(n=30)$ had a high total blood cholesterol. The numbers were slightly better in diabetic patients with $79 \%(n=158)$ having normal total cholesterol and $21 \%(n=42)$ had a high total blood cholesterol. The difference between cases and controls was not statically significant (odds ratio $=1.6$ ) (univariate analysis $P$-value $=0.09)($ multivariate analysis $P$-value $=0.51)($ Table 4$)$.

\section{Triglyceride}

Triglyceride also followed the same trend as total cholesterol. Among diabetic patients, $31.5 \%(n=63)$ had a high triglyceride and $68.5 \%(n=137)$ had a normal triglyceride. The percentages were slightly high in DFD patients $43 \%(n=43)$ who have high triglyceride and $57 \%(n=57)$ had controlled level. Triglyceride showed a trend to significant correlation with DFD. (odds ratio $=1.6$ ) (univariate analysis $P$-value $=0.06$ ) (multivariate analysis $P$-value $=0.80)($ Table 4$)$.

\section{$H D L$}

Percentages of HDL were the same in both cases and controls with $92 \%$ having a normal HDL level in the blood and $8 \%$ had low HDL levels. HDL did not show any association with DFD (odds ratio $=1$ ) (univariate analysis $P$-value $=1$ ) (multivariate analysis $P$-value $=0.55)$ (Table 4$)$.

\section{LDL}

LDL did not show any association with the progression of DFD. Sixty-three percent $(63 \%)(n=126)$ of diabetic patient had a normal triglyceride level while $37 \%(n=74)$ had a high triglyceride level. On the other side, $42 \%(n=42)$ of DFD patients had a high level of triglyceride and $58 \%(n=58)$ were within the normal limits. LDL did not show a statistically significant correlation with DFD (odds ratio $=1.2$ ) (univariate analysis $P$-value $=0.40)($ multivariate analysis $P$-value $=0.87)$ (Table 4).

\section{Multiple risk factors}

Eighty-six percent $(86 \%)$ of DFD patients had two or more significant risk factors; and only $14(14 \%)$ of them got one or no significant risk factor. On the other hand, 70 (35\%) of diabetic patients had two or more significant risk factors and 130 (65\%) got one or no significant risk factor. Multivariate analysis showed that those who had two or more significant risk has a 10 times higher risk of developing DFD in comparison to those who do not have any of these risk factors (Table 5).

\section{Table 5 . The effect of multiple risk factors on the development} of DFD.

\begin{tabular}{lccc}
\hline DFD & Yes & No & P value \\
\hline Exposed & $86(86 \%)$ & $70(35 \%)$ & \\
Not exposed & $14(14 \%)$ & $130(65 \%)$ & $<0.001$ \\
\hline
\end{tabular}

*Exposed= two or more significant risk factors

\section{Discussion}

DFD progression and risk factors have been investigated in many previous studies. This study has shed the light on nine different risk factors and identified their correlation with the presence of DFD. Three of the risk factors were found to have a statistically significant correlation with DFD. Poorly controlled blood glucose (HbA1c) was shown to be a strong contributing risk factor with DFD. This finding is in line with many other studies. ${ }^{14-17}$ Our study found a strong correlation between $\mathrm{HbA} 1 \mathrm{C}$ and DFD, and this is probably due to the effect of $\mathrm{HbA1c}$ on the development of neuropathy and microvascular injury. ${ }^{18}$ $\mathrm{BMI}$ is also a common risk factor for DFD but it's impact is less than that of HbAlc. Many studies demonstrated an association between BMI and DFD. ${ }^{6,12,14}$ Our study revealed that having a BMI of $>30$ has a significant correlation with the development of DFD which could be due to external or internal influence of the increased weight on the presence of foot ulcers. ${ }^{19,20}$

Furthermore, our study confirmed that increased blood pressure has a strong association with the development of DFD. This finding was also supported by many other studies. ${ }^{12,15}$ This could be due to the occlusion of the blood vessels in the limb. ${ }^{21}$

Gender is a very controversial subject in DFD progression, many studies showed that males are likely to develop DFD while others did not confirm this finding. ${ }^{22}$ In our study, we did not find any correlation between gender and DFD. Based on our results, it was obvious that the general control (blood glucose level $\mathrm{HbAlc}$, blood pressure and BMI) in females was worse than males and this could play a very important role in developing DFD, but at the same time, males are more prone to foot ulcers due to probably physical activity and less foot care in comparison to females. It was found that males with DFD were slightly more than females but the difference was not statistically significant. Larger sample size is required to confirm this finding.

Age at onset of DM was also studied and it did not show any correlation with the occurrence of DFD. Most of the studies showed that longer duration of diabetes increases the chance of getting diabetes complications which means those who got diabetes at a younger age have a higher chance of getting manifestations later in life. ${ }^{12}$ Some studies demonstrated that age of diabetic patients does not play an important role in DFD development. ${ }^{14,23}$

This study revealed that lipid profile do not have any correlation with DFD. Our finding is similar to many other studies. ${ }^{14,23}$ Lipid profile in general do not appear to play an important role in developing DFD.

Multivariate analysis of the risk factors showed a strong association with the development of DFD for those who had two or more risk factors. It has been found that patients with two or more significant risk factors have 11 times higher risk of DFD in comparison to those who have less or no risk factors.

\section{Limitations}

Being of a retrospective nature, this is one of the limitations of this study and therefore limited our ability to analyze some risk factors, which was intended to be studied initially. In addition, direct access to AlShifa system is available only for the past 5 years. Furthermore, larger sample size is required to confirm these findings. 


\section{Recommendations}

Prevention and control of DM and DFD requires that it be prioritized at the national and governorate levels while strengthening multisectoral, legislative and societal aspects, as it increases risks to health, social, and financial progress. Second, encourage large-scale awareness campaigns to control modifiable risk factors for people with diabetes: physical inactivity, unhealthy diet, and alcohol consumption. Third, improve the health system to ensure access to basic techniques for testing, diagnosing, valid, and safe drugs as well as continuous monitoring of diabetes in primary health-care sessions. Fourth, implement evidence-based practices and decision-making in diabetes management through the use of a simplified information system to ensure the best quality of data. Fifth, developing health clinics in all health institutes to promote early detection and examination of diabetes, as well as acting as data sources for prevention and health progress. Sixth, improving the implementation of the Omani national policy on diet, physical activity, and health, and engaging with the agricultural sector to promote healthy diets and eating habits. Seventh, strengthening laws related to the manufacture, packaging, and marketing of food and beverages to reduce consumption of unhealthy foods. Eighth, encouraging an active lifestyle and reducing the sedentary lifestyle through implementing the physical activity toolkit in the country.

\section{Conclusion}

DFD is a real-life threating complication of diabetes. Poorly controlled diabetes $(\mathrm{HbAlc}>7)$, obesity $\left(\mathrm{BMI}>30 \mathrm{~kg} / \mathrm{m}^{2}\right)$ and high blood pressure $(>140 / 90 \mathrm{mmHg})$ were significantly correlated with progression to DFD. These risk factors are modifiable, and so it is important to raise the level of awareness among diabetic patients. Controlling these risk factors can help in reducing future DFD related morbidity and mortality. There are few studies with low output and impact in the fields of diabetic foot care and DFD in Oman which focused on epidemiology, prevention, and management of DFD. More studies are required to further improve our understanding of the scope of DFD, and the impact of the existing diabetes programs on the level of diabetic foot care in Oman. Continuous support governmental and financial support for diabetic foot research is important. Cooperation with major regional and international diabetic foot research organizations, such as The 'Gulf Diabetic Foot Working Group' (GDFWG) and the 'International Working Group on the Diabetic Foot', is essential (i.e., through sharing intervention, programs, and initiating collaborative multicenter studies).

There is a dearth of studies related to DFD in Oman, thus more studies are urgently needed, and stronger screening programs are required to obtain the exact number of DFD patients in Oman who are at high risk. The investigated results are essential to support the formulation and implementation of initiation and action plans for DM and DFD that enhance patients' health status. Developing this plan can ensure not only a huge financial return on investment in the health budget but more importantly, improving the quality of life for Omanis.

\section{Conflict of interest}

The authors declare that they have no conflict of interest.

\section{References}

1. Al-Mawali A. Non-communicable diseases: shining a light on cardiovascular disease, Oman's biggest killer. Oman Med J [Internet]. 2015 Jul;30(4):227-8. Available from: https://pubmed.ncbi.nlm.nih.gov/26366254

2. World Health Organization. Diabetes Mellitus WHO [Internet]. Definition, Diagnosis and Classification of Diabetes Mellitus and its Complications. 1999. p. 49. Available from: https://www.staff.ncl.ac.uk/philip.home/ who_dmg.pdf

3. International Diabetes Federation. IDF DIABETES ATLAS [Internet]. 2017 1-150 p. Available from: http://www.diabetesatlas.org/resources/2017atlas.html

4. Annual health report $2011 \mathrm{MoH}$ [Internet]. MOH. 2011. Available from: https://www.moh.gov.om/en/web/statistics/-/201-6

5. Annual report MoH 2016 [Internet]. 2016. p. 81-2. Available from: https:// www.moh.gov.om/en/web/statistics/-/20-46

6. Almobarak AO, Awadalla H, Osman M, Ahmed MH. Prevalence of diabetic foot ulceration and associated risk factors: an old and still major public health problem in Khartoum, Sudan? Ann Transl Med. 2017 Sep;5(17):340.

7. Alexiadou K, Doupis J. Management of diabetic foot ulcers. Diabetes Ther. 2012;3(1):1-15.

8. Alkhier Ahmed D, Elsharief E, Alsharief A. The Diabetic Foot in the Arab World. J Diabet Foot Complicat. 2011;3(3):55-61.

9. Annual health report $2003 \mathrm{MoH}$ [Internet]. 2002. Available from: https:// www.moh.gov.om/documents/274609/1595174/2002/التقريرالسنوي+الإحصائي 7f90306b-27bc-49d6-9c20-9f831a11e78c

10. Annual health report $2013 \mathrm{MoH}$ [Internet]. 2003. Available from: https:// www.moh.gov.om/en/web/statistics/annual-reports?p_p_id=122 INSTANCE_TruTPmhSILZ9\&p_p_lifecycle=0\&p_p_state=normal\&p_p_ mode $=$ view\&p_p_col_id =column-1\&p_p_col_pos $=1 \& p \_p \_c o l \_$ count=2\&p_r_p_564233524_resetCur=true\&p_r_p_564233524_ categoryld $=274760$

11. Al-Busaidi IS, Abdulhadi NN, Coppell KJ. Care of patients with diabetic foot disease in Oman. Vol. 16, Sultan Qaboos Univ Med J. 2016:e270-6.

12. Al-Rubeaan K, Al Derwish M, Ouizi S, Youssef AM, Subhani SN, Ibrahim $\mathrm{HM}$, et al. Diabetic foot complications and their risk factors from a large retrospective cohort study. PLoS One [Internet]. 2015;10(5). Available from: http://dx.doi.org/10.1371/journal.pone.0124446

13. Al-Busaidi IS. Diabetic foot disease in Oman: a call for more research. Oman Med J. 2017;32(4):354-5

14. Nyamu PN, Otieno CF, Amayo EO, Mcligeyo SO. Risk factors and prevalence of diabetic foot ulcers at Kenyatta National Hospital, Nairobi Background: Diabetic foot ulcers contribute significantly to the morbidity and mortality of patients with diabetes mellitus. The diabetic patients with foot ulce. East Africa Med J. 2003;80(1):36-43

15. Fawzy MS, Alshammari MA, Alruwaili AA, Alanazi RTR, Alharbi JAM, Almasoud AMR, et al. Factors associated with diabetic foot among type 2 diabetes in Northern area of Saudi Arabia: a descriptive study. BMC Res Notes. 2019 Jan;12(1):51

16. Dubský M, Jirkovská A, Bem R, Fejfarová V, Skibová J, Schaper NC, et al. Risk factors for recurrence of diabetic foot ulcers: prospective follow-up analysis in the Eurodiale subgroup. Int Wound J [Internet]. 2013;10(5):555-61. Available from: https://doi.org/10.1111/j.1742-481X.2012.01022.X

17. Lavery LA, Armstrong DG, Wunderlich RP, Mohler MJ, Wendel CS, Lipsky BA. Risk factors for foot infections in individuals with diabetes. Diabetes Care [Internet]. 2006 Jun 1;29(6):1288 LP - 1293. Available from: http://care. diabetesjournals.org/content/29/6/1288.abstract

18. Nabil Abd El Fatah EA etc. Study of risk factors of diabetic foot ulcers. 2014;28-34. Available from: www.mmj.eg.net/article.asp?issn=11102098; year $=2014 ;$ volume $=27 ;$ issue $=1 ;$ spage $=28$; epage $=34$; aulast $=A l ;$ aid $=$ M enoufiaMedJ_2014_27_1_28_132298\%0D

19. Boyko EJ, Ahroni JH, Stensel V, Forsberg RC, Davignon DR, Smith DG. A prospective study of risk factors for diabetic foot ulcer. The Seattle Diabetic Foot Study. Diab Care [Internet]. 1999 Jul 1;22(7):1036 LP - 1042. Available from: http://care.diabetesjournals.org/content/22/7/1036.abstract 
20. Mantey I, Foster A V, Spencer S, Edmonds ME. Why do foot ulcers recur in diabetic patients? Diab Med. 1999 Mar;16(3):245-9.

21. Ogbuawa O, Williams JT, Henry WLJ. Diabetic gangrene in black patients. South Med J. 1982 Mar;75(3):285-8.

22. Dinh $T$, Veves $A$. The influence of gender as a risk factor in diabetic foot ulceration. Vol. 20, Wounds : a compendium of clinical research and practice. 2015. 127-131 p.
23. Manda V, Sreedharan J, Muttappallymyalil J, Das R, Hisamatsu E. Foot ulcers and risk factors among diabetic patients visiting Surgery Department in a University Teaching Hospital in Ajman, UAE. Int J Med Public Heal. 2012;2(3):34-8.

This work is licensed under a Creative Commons Attribution-NonCommercial 3.0 Unported License which allows users to read, copy, distribute and make derivative works for non-commercial purposes from the material, as long as the author of the original work is cited properly. 